Ruslan Pak ${ }^{1}$, Olena Lanets ${ }^{2}$

1. Department of engineering mechanics (armament and technics of engineering army), Hetman Petro Sahaidachnyi National Army Academy, Ukraine, Lviv, Heroes of Maidan street, 32, , E-mail: rpak@ukr.net 2. Department of Technical Mechanics and Dynamics of Machines, Lviv Polytechnic National University, Ukraine, Lviv, S. Bandery street, 12, E-mail: lanecolena@gmail.com

\title{
CALCULATION OF WAVE FIELDS IN A LAYERED HALF-SPACE WITH ABSORPTION BASED ON THE THOMSON-HASKELL METHOD
}

Received: November 8, 2018 / Revised: December 24, 2018 / Accepted: December 26, 2018

(C) Pak R., Lanets O., 2018

Abstract. This article is devoted to the development of calculation method of seismic waves on the free surface of a horizontally-layered half-space, which are perturbed by local sources. Previous obtained relations for scalar potentials of direct waves P, SV and SH from the simple force in a homogeneous environment are used for this purpose. It let us derive formulas for a complete wave field in the horizontally-layered isotropic elastic half-space, which is perturbed by the point source in the form of the simple time-depend force by using modified Thomson-Haskell's matrix method. Exact expressions for three-dimensional displacement vector on the free surface are developed. Obtained results are generalized on the case of absorbing environments.

The algorithm is built and the computer program for calculation of three-component synthetic seismograms in the horizontally-layered isotropic environment with absorption is written on the base of developed method of direct problem solving.

In order to verify the efficiency and stability of the algorithm, full synthetic seismograms were calculated on the test examples.

Keywords: mathematical modelling, seismic wave field, Thompson-Haskell method, horizontally-layered environment, seismic quality factor, tensor of seismic moment, synthetic seismogram, local resonance.

\section{Introduction}

Mathematical modelling of wave fields is an effective tool, which is used particularly for seismic image environment construction, for inversion of seismic data for determination of the earthquake source parameters, for design of the observation systems, for theoretical investigations of the wave processes.

Processing and interpretation of seismic data, with the aim to determine the parameters of the environment or source, are based on the use of registered wave field and iterative process of refinement, on each stage of which the direct problem is solving. The direct problem (wave field synthesis) can be solved with the use of mathematical modelling. The environmental parameters can vary in all directions, so 3Dmodeling requires significant resources of the computer time and large amount of RAM. So, these methods are difficult to be used for solving of the inverse problems because the multiple wave field synthesis is required for this. In addition, only full wave fields can be calculated by these universal methods and in many cases it is desirable to have a certain definite part of this field. Therefore, the wave field synthesis by matrix method is still widely used. It has the unique properties. In particular this is considering of the effects of anisotropy, absorption, availability of free surface and curved limits of separation, ability to specify arbitrary types of sources, getting of the part of the wave field which contains the required types of the waves. Additionally, the matrix method is often used for inversion of seismic moment tensor. However, during the direct computer implementation of the method, it is necessary to find sums of the growing and falling exponents, what leads to the loss of calculation accuracy [1]. 


\section{Calculation of Wave Fields in a Layered Half-space with Absorption based on the ...}

\section{Review of Modern Information Sources on the Subject of the Paper}

It was suggested a number of methods to eliminate this disadvantage. For example, the use of minors instead of propagators [2], the transition to matrixes of the high order [1], application of recursive formulas for calculating minors considering different behaviour of exponents depending on the wave number [3], recursive algorithm for calculation of reflection matrix and refractive matrix at each boundary [4]. Recursive algorithm is the part of the reflectivity method [5], and discrete wavenumber method [6].

In this regard, it is the necessity to develop the methods of mathematical modelling, which would allow to calculate quickly and accurately all components of the wave fields, generated by the sources of different types. In this paper the method of mathematical modelling of wave field on the free surface of the horizontally-layered half-space from a point source is offered. Here the approach, described in the monograph [1] is generalized for the other types of wave sources (simple force and couple) and its location in any layer of horizontally-layered half-space at the presence of absorption. The test results of the algorithm and program for calculating seismograms based on method are also described in the article.

\section{Objectives and Problems of Research}

Let's consider horizontally-layered half-space in a cylindrical coordinate system $(r, \square, z)$ which consists of $(n+1)$ homogeneous, isotropic, linear-elastic layers with flat boundaries of the division. The origin of the coordinate system is on the free surface of this environment and the axis $O z$ is directed downwards towards the layers. Assume that on the layers boundaries, which are characterized by thickness, density, velocity of longitudinal and transverse spreading of waves $h_{i}, \rho_{i}, v_{P i}, v_{S i}$ respectively $(i=1,2, \ldots, n+1)$, the conditions of rigid contact are executed. It is considered that $h_{n+1}=+\infty$ is for $(n+1)$ layer. Let the arbitrary layer at the moment of time $t=0$ the point source begins to act as a concentrated force $\mathbf{f}(t)=\left(f_{r}(t), f_{\phi}(t), f_{z}(t)\right)^{T}$. This layer let be imaginary divided by horizontal boundary passing through the point of the application of force. Thus, two layers are formed, the top of which will have number $s$, and the bottom layer will be numbered $s+1$. Then the source is situated on the $s$ boundary at the point $\left(0,0, z_{s}\right)$.

Elastic wave propagation in every layer described by differential equation [7]

$$
\rho_{i} \ddot{\mathbf{u}}_{i}=\left(\lambda_{i}+2 \mu_{i}\right) \nabla\left(\nabla \cdot \mathbf{u}_{i}\right)-\mu_{i} \nabla \times\left(\nabla \times \mathbf{u}_{i}\right),
$$

where $\mathbf{u}_{i}=\mathbf{u}_{i}(\mathbf{r}, t)$ is displacement vector, $\rho_{i}$ is density, $\lambda_{i}$ and $\mu_{i}$ are Lame parameters for $i$-th layer. It is assumed that the intensity of source $\mathbf{f}(t)$ for $t<0$ becomes zero, so formed wave field must satisfy initial zero conditions:

$$
\dot{\mathbf{u}}_{i}(\mathbf{r}, 0)=0, \mathbf{u}_{i}(\mathbf{r}, 0)=0 .
$$

Wave field must also satisfy boundary conditions:

$$
\begin{gathered}
\left.\mathbf{u}_{i}\right|_{z=H_{i}}=\left.\mathbf{u}_{i+1}\right|_{z=H_{i}},\left.\tau_{z l i}\right|_{z=H_{i}}=\left.\tau_{z l i+1}\right|_{z=H_{i}} \text { when } \mathbf{r} \neq\left(0,0, z_{s}\right),(i=1,2, \ldots, n) ; \\
\left.\tau_{z l}\right|_{z=0}=0 ; \\
{\left.\left[\tau_{z l}(\mathbf{r}, t)\right]\right|_{\mathbf{r}=\left(0,0, z_{s}\right)}=-\frac{f_{l}(t) \delta(r)}{2 \pi r} .}
\end{gathered}
$$

It is assumed that radiation condition is given : the waves from the half-space are not returning back. By the symbol $\left[\tau_{z l}(\mathbf{r}, t)\right]$ the jump $\tau_{z l}\left(z_{s}+0\right)-\tau_{z l}\left(z_{s}-0\right)$ of function $\tau_{z l}(\mathbf{r}, t)$ for $z=z_{S}, l=\{r, \phi, z\}$ is marked. In formulas (3) stress tensor components for a given layer marked by letter $\tau$ with indexes, $H_{i}$ is the distance from the free surface to the bottom boundary of $i$-layer, $\delta(r)$ is Dirac function.

There is a potential of field of displacements $\varphi_{i}, \psi_{i}, \chi_{i}$ for equation (1), [7], which related to the components of the displacement vector and stress tensor elements by formulas: 


\section{Ruslan Pak, Olena Lanets}

$$
\begin{gathered}
\mathbf{u}_{i}=\nabla \cdot \varphi_{i}+\nabla \times\left(\nabla \times \mathbf{k} \Psi_{i}\right)+\nabla \times \mathbf{k} \chi_{i} ; \\
\tau_{r z i}=\mu_{i}\left(2 \varphi_{i, r z}+2 \Psi_{i, z z r}+\frac{1}{r} \chi_{i, \phi z}-\frac{1}{v_{S i}^{2}} \Psi_{i, t t r}\right) ; \\
\tau_{z \phi i}=\frac{\mu_{i}}{r}\left[2 \varphi_{i, z \phi}+2 \Psi_{i, z z \phi}-r \chi_{i, r z}-\frac{1}{v_{S i}^{2}} \Psi_{i, t t \phi}\right] ; \\
\tau_{z z i}=\frac{\lambda_{i}}{v_{p i}^{2}} \varphi_{i, t t}+2 \mu_{i}\left(\varphi_{i, z z}+\Psi_{i, z z z}-\frac{1}{v_{S i}^{2}} \Psi_{i, t t z}\right) .
\end{gathered}
$$

These potentials satisfy the wave equation:

$$
\ddot{\varphi}_{i}=v_{P i}^{2} \Delta \varphi_{i}, \quad \ddot{\Psi}_{i}=v_{S i}^{2} \Delta \Psi_{i}, \quad \ddot{\chi}_{i}=v_{S i}^{2} \Delta \chi_{i} .
$$

After introducing transformation

$$
Y=\Xi(\tilde{Y})=-\frac{1}{4 \pi \rho} \int_{0}^{\infty} \frac{d k}{2 \pi j} \int_{\sigma-j \infty}^{\sigma+j \infty} \tilde{Y} \exp (k t \eta) d \eta, j=\sqrt{-1},
$$

the relationship for images of potentials for direct (primary) waves $\mathrm{P}, \mathrm{SV}$ and $\mathrm{SH}$, perturbed by a given source in the layer number s, respectively $\tilde{\varphi}_{s}^{u}, \tilde{\Psi}_{s}^{u}, \tilde{\chi}_{s}^{u}$ and in the layer with number $s+1$ respectively $\tilde{\varphi}_{s+1}^{d}, \tilde{\Psi}_{s+1}^{d}, \tilde{\chi}_{s+1}^{d}$ can be written. The image of potentials for a homogeneous isotropic environment, obtained in article [8], is used for this. Considering that the point source is in the layer with number s, we get:

$$
\begin{gathered}
\tilde{\varphi}_{s}^{u}=J_{1}(k r) F_{r}(k \eta) X_{s}^{(1)+} e^{k \alpha_{s}\left(z-H_{s-1}\right)}+J_{0}(k r) F_{z}(k \eta) \hat{X}_{s}^{(2)+} e^{k \alpha_{s}\left(z-H_{s-1}\right)} ; \\
\tilde{\varphi}_{s+1}^{u}=J_{1}(k r) F_{r}(k \eta) X_{s+1}^{(1)-} e^{k \alpha_{s+1}\left(H_{s}-z\right)}+J_{0}(k r) F_{z}(k \eta) X_{s+1}^{(2)-} e^{k \alpha_{s+1}\left(H_{s}-z\right)} ; \\
\tilde{\Psi}_{s}^{u}=k^{-1} J_{1}(k r) F_{r}(k \eta) Y_{s}^{(1)+} e^{k \beta_{s}\left(z-H_{s-1}\right)}+k^{-1} J_{0}(k r) F_{z}(k \eta) Y_{s}^{(2)+} e^{k \beta_{s}\left(z-H_{s-1}\right)} ; \\
\tilde{\Psi}_{s+1}^{d}=k^{-1} J_{1}(k r) F_{r}(k \eta) Y_{s+1}^{(1)-} e^{k \beta_{s+1}\left(H_{s}-z\right)}+k^{-1} J_{0}(k r) F_{z}(k \eta) Y_{s+1}^{(2)-} e^{k \beta_{s+1}\left(H_{s}-z\right)} ; \\
\tilde{\chi}_{s}^{d}=J_{1}(k r) F_{\varphi}(k \eta) Z_{s}^{+} e^{k \beta_{s}\left(z-H_{s-1}\right)} ; \\
\tilde{\chi}_{s+1}^{d}=J_{1}(k r) F_{\varphi}(k \eta) Z_{s+1}^{-} e^{k \beta_{s+1}\left(H_{s}-z\right)} ;
\end{gathered}
$$

where

$$
\begin{aligned}
& X_{S}^{(1)+}=\frac{e^{-k \alpha_{s} h_{s}}}{\alpha_{s} \eta^{2}} ; X_{S}^{(2)+}=-\frac{e^{-k \alpha_{s} h_{s}}}{\eta^{2}} ; X_{s+1}^{(1)-}=\frac{1}{\alpha_{s+1} \eta^{2}} ; X_{s+1}^{(2)-}=\frac{1}{\eta^{2}} ; Y_{s}^{(1)+}=-\frac{e^{-k \beta_{s} h_{s}}}{\eta^{2}} ; \\
& Y_{s}^{(2)+}=\frac{e^{-k \beta_{s} h_{s}}}{\beta_{s} \eta^{2}} ; Y_{s+1}^{(1)-}=\frac{1}{\eta^{2}} ; Y_{s+1}^{(2)-}=\frac{1}{\beta_{s+1} \eta^{2}} ; Z_{s}^{+}=\frac{\left(\beta_{s}^{2}-1\right) e^{-k \beta_{s} h_{s}}}{\beta_{S} \eta^{2}} ; \\
& Z_{s+1}^{-}=\frac{\left(\beta_{s+1}^{2}-1\right) e^{-k \beta_{s+1} h_{s}}}{\beta_{s+1} \eta^{2}} .
\end{aligned}
$$

In formulas (7) and (8) $\alpha_{s}=\sqrt{1+\eta^{2} / v_{S S}^{2}}, \beta_{s}=\sqrt{1+\eta^{2} / v_{P S}^{2}}, H_{s}=z_{s}, J_{0}(k r), J_{1}(k r)$ are Bessel functions of order 0 and $1, F_{r}(k \eta), F_{\phi}(k \eta), F_{z}(k \eta)$ are images of functions respectively $f_{r}(t)$, $f_{\phi}(t), f_{z}(t)$ by Laplace integral $F(k \eta)=\int_{0}^{\infty} f(t) \exp (-k \eta t) d t$. In order to reduce the expression, we will write these functions without arguments. 


\section{Calculation of Wave Fields in a Layered Half-space with Absorption based on the ...}

\section{Main Material Presentation}

Solution based on the Thomson-Haskell method.

The energy of primary waves is redistributed in the conditions of inhomogeneous half-space. Therefore, the solution of the wave equations (5) for every layer of the environment should be sought taking the initial conditions (2) into account, in the form of solutions (7) for a homogeneous space:

$$
\begin{gathered}
\tilde{\varphi}_{i}=J_{1} X_{i}^{(1)} F_{r}+J_{0} X_{i}^{(2)} F_{z} ; \\
\tilde{\Psi}_{i}=k^{-1} J_{1} Y_{i}^{(1)} F_{r}+k^{-1} J_{0} Y_{i}^{(2)} F_{z} ; \\
\tilde{\chi}_{i}=J_{1} Z_{i} F_{\phi},
\end{gathered}
$$

where

$$
\begin{gathered}
X_{i}^{(1)}=X_{i}^{(1)+} e^{k\left(z-H_{i-1}\right) \alpha_{i}}+X_{i}^{(1)-} e^{k\left(H_{i-1}-z\right) \alpha_{i}} ; X_{i}^{(2)}=X_{i}^{(2)+} e^{k\left(z-H_{i-1}\right) \alpha_{i}}+X_{i}^{(2)-} e^{k\left(H_{i-1}-z\right) \alpha_{i}} ; \\
Y_{i}^{(1)}=Y_{i}^{(1)+} e^{k\left(z-H_{i-1}\right) \beta_{i}}+Y_{i}^{(1)-} e^{k\left(H_{i-1}-z\right) \beta_{i}} ; Y_{i}^{(2)}=Y_{i}^{(2)+} e^{k\left(z-H_{i-1}\right) \beta_{i}}+Y_{i}^{(2)-} e^{k\left(H_{i-1}-z\right) \beta_{i}} \\
Z_{i}=Z_{i}^{+} e^{k\left(z-H_{i-1}\right) \beta_{i}}+Z_{i}^{-} e^{k\left(H_{i-1}-z\right) \beta_{i}} .
\end{gathered}
$$

These formulas contain magnitudes (amplitudes of potentials) $X_{i}^{(1)+}, X_{i}^{(2)+}, Y_{i}^{(1)+}, Y_{i}^{(2)+}, Z_{i}^{+}$ describing waves in the $i$-th layer spreading upwards (in the opposite direction of the Oz axis) and $X_{i}^{(1)-}$, $X_{i}^{(2)-}, Y_{i}^{(1)-}, Y_{i}^{(2)-}, Z_{i}^{-}$describing waves spreading downwards (in the direction of the Oz axis). These amplitudes of potentials are the functions of variables $k$ and $\eta$.

After substituting of the relation (9) into (6), the resulting potentials will be substituted into the formulas (4) and the expressions for the components of the displacement vector and elements of the stress tensor in each layer will be obtained:

$$
\begin{gathered}
\tilde{u}_{r i}=k^{2} J_{0} U_{r i}^{(1)} F_{r}-k^{2} J_{1} U_{r i}^{(2)} F_{z}-\frac{k}{r} J_{1}\left(U_{r i}^{(2)}+U_{\phi i}\right) F_{r} ; \\
\tilde{u}_{\phi i}=-k^{2} J_{0} U_{\phi i} F_{\phi}+\frac{k}{r} J_{1}\left(U_{r i}^{(1)}+U_{\phi i}\right) F_{\phi} ; \\
\tilde{u}_{z i}=k^{3} J_{1} U_{z i}^{(1)} F_{r}+k^{3} J_{0} U_{z i}^{(2)} F_{z} ; \\
\tilde{\tau}_{r z i}=k^{3} J_{0} T_{r z i}^{(1)} F_{r}-k^{3} J_{1} T_{r z i}^{(2)} F_{z}-\frac{k^{2}}{r} J_{1}\left(T_{r z i}^{(1)}+T_{z \phi i}\right) F_{r} ; \\
\tilde{\tau}_{z \phi i}=-k^{3} J_{0} T_{z \phi i} F_{\phi}+\frac{k^{2}}{r} J_{1}\left(T_{r z i}^{(1)}+T_{z \phi i}\right) F_{\phi} ; \\
\tilde{\tau}_{z z i}=k^{3} J_{1} T_{z z i}^{(1)} F_{r}+k^{3} J_{0} T_{z z i}^{(2)} F_{z} .
\end{gathered}
$$

Let's write the expressions for functions $U_{r i}^{(1)}, U_{r i}^{(2)}, U_{\phi i}, U_{z i}^{(1)}, U_{z i}^{(2)}, T_{r z i}^{(1)}, T_{r z i}^{(2)}, T_{z \phi i}, T_{z z i}^{(1)}, T_{z z i}^{(2)}$ in the form of matrix equations:

where

$$
\begin{gathered}
\mathbf{W}_{i}^{(1)}\left(H_{i}\right)=\mathbf{A}_{i} \mathbf{L}_{i} \mathbf{Z}_{i}^{(1)} ; \mathbf{W}_{i}^{(2)}\left(H_{i}\right)=\mathbf{A}_{i} \mathbf{L}_{i} \mathbf{Z}_{i}^{(2)} ; \mathbf{W}_{i}^{*}\left(H_{i}\right)=\mathbf{A}_{i}^{*} \mathbf{L}_{i}^{*} \mathbf{Z}_{i}^{*} ; \\
\mathbf{W}_{i+1}^{(1)}\left(H_{i}\right)=\mathbf{A}_{i+1} \mathbf{Z}_{i+1}^{(1)} ; \mathbf{W}_{i+1}^{(2)}\left(H_{i}\right)=\mathbf{A}_{i+1} \mathbf{Z}_{i+1}^{(2)} ; \mathbf{W}_{i+1}^{*}\left(H_{i}\right)=\mathbf{A}_{i+1}^{*} \mathbf{z}_{i+1}^{*},
\end{gathered}
$$

$$
\begin{gathered}
\mathbf{W}_{i}^{(1)}=\left(U_{r i}^{(1)}, U_{z i}^{(1)}, T_{r z i}^{(1)}, T_{z z i}^{(1)}\right)^{\mathrm{T}} ; \mathbf{W}_{i}^{(2)}=\left(U_{r i}^{(2)}, U_{z i}^{(2)}, T_{r z i}^{(2)}, T_{z z i}^{(2)}\right)^{\mathrm{T}} ; \mathbf{W}_{i}^{*}=\left(U_{\phi i}, T_{z \phi i}\right)^{\mathrm{T}} ; \\
\mathbf{Z}_{i}^{(1)}=\left(X_{i}^{(1)+}, X_{i}^{(1)-}, Y_{i}^{(1)+}, Y_{i}^{(1)-}\right)^{\mathrm{T}} ; \mathbf{Z}_{i}^{(2)}=\left(X_{i}^{(2)+}, X_{i}^{(2)-}, Y_{i}^{(2)+}, Y_{i}^{(2)-}\right)^{\mathrm{T}}
\end{gathered}
$$




$$
\mathbf{Z}_{i}^{*}=\left(Z_{i}^{+}, Z_{i}^{-}\right)^{\mathrm{T}} ; \mathbf{A}_{i}^{*}=\left(\begin{array}{cc}
1 & 1 \\
\mu_{i} \beta_{i} & -\mu_{i} \beta_{i}
\end{array}\right) ; \mathbf{L}_{i}^{*}=\left(\begin{array}{cc}
e^{k \beta_{i} h_{i}} & 0 \\
0 & e^{-k \beta_{i} h_{i}}
\end{array}\right) .
$$

The matrices $\mathbf{A}_{i}, \mathbf{L}_{i}$ are given in [1]. We also will write the relation (8) in the form of components of the vectors:

$$
\begin{gathered}
\widehat{\mathbf{Z}}_{s}^{(1)}=\frac{1}{\eta^{2}}\left(\frac{e^{-k \alpha_{s} h_{s}}}{\alpha_{s}}, 0,-e^{-k \beta_{s} h_{s}}, 0\right)^{\mathrm{T}} ; \widehat{\mathbf{Z}}_{s+1}^{(1)}=\frac{1}{\eta^{2}}\left(0, \frac{1}{\alpha_{s+1}}, 0,1\right)^{\mathrm{T}} ; \\
\hat{\mathbf{Z}}_{s}^{(2)}=\frac{1}{\eta^{2}}\left(-e^{-k \alpha_{s} h_{s}}, 0, \frac{e^{-k \beta_{s} h_{s}}}{\beta_{s}}, 0\right)^{\mathrm{T}} ; \hat{\mathbf{Z}}_{s+1}^{(2)}=\frac{1}{\eta^{2}}\left(0,1,0, \frac{1}{\beta_{s+1}}\right)^{\mathrm{T}} ; \\
\hat{\mathbf{Z}}_{s}^{*}=\frac{1}{\eta^{2}}\left(\frac{\beta_{s}^{2}-1}{\beta_{s}} e^{-k \beta_{s} h_{s}}, 0\right)^{\mathrm{T}} ; \hat{\mathbf{Z}}_{s+1}^{*}=\frac{1}{\eta^{2}}\left(0, \frac{\beta_{s+1}^{2}-1}{\beta_{s+1}}\right)^{\mathrm{T}} .
\end{gathered}
$$

For considering the processes of reflection and passing on the boundaries of the layers we will use a matrix method, according to which the expressions for matrices, which characterize the layers and halfspace will be established. For this purpose to every environment and every separating boundary corresponds (at the level of the functions under integral of the Mellin's integrals) characteristic matrix, and the waves propagation process is taken into account by the production of such matrices in a certain order. This approach, which corresponds to the Thomson-Hasskel method, was described in the monograph [1].

Let us consider the first equality from formulas (11) and (12). Using the conditions for ideal contact between the layers $\mathbf{W}_{i+1}^{(1)}\left(H_{i}\right)=\mathbf{W}_{i}^{(1)}\left(H_{i}\right)$, and taking into account that the wave field in the layers $s$ and $s+1$ is the sum of the direct wave from the source and the waves generated by the interaction of the direct wave with the environment over the source and below it, we obtain:

$$
\mathbf{Z}_{n+1}^{(1)}=\mathbf{D}_{s+1}^{n+1} \mathbf{S}^{(1)}+\mathbf{D} \mathbf{W}_{1}^{(1)}(0)
$$

where $\mathbf{W}_{1}^{(1)}(0)=\left(U_{r 0}^{(1)}, U_{z 0}^{(1)}, 0,0\right)^{\mathrm{T}}$ is column vector of functions-images of the displacements and stresses on a free surface, $\mathbf{S}^{(1)}=\left(s_{p}^{(1)}\right)=\frac{1}{\eta^{2}}\left(-\frac{1}{\alpha_{s}}, \frac{1}{\alpha_{s}}, 1,1\right)^{\mathrm{T}}$. According to the condition of radiation we have $\mathbf{Z}_{n+1}^{(1)}=\left(0, X_{n+1}^{(1)-}, 0, Y_{n+1}^{(1)-}\right)^{\mathrm{T}}, \quad \mathbf{D}=\mathbf{A}_{n+1}^{-1} \mathbf{C}_{n} \ldots \mathbf{C}_{1}, \quad \mathbf{D}_{s+1}^{n+1}=\mathbf{A}_{n+1}^{-1} \mathbf{C}_{n} \ldots \mathbf{C}_{s+2} \mathbf{A}_{s+1} \mathbf{L}_{s+1}, \quad\left(\mathbf{C}_{i}=\mathbf{A}_{i} \mathbf{L}_{i} \mathbf{A}_{i}^{-1} \quad\right.$ is layer matrix). The matrices $\mathbf{D}=\left(d_{l p}\right)$ and $\mathbf{D}_{s+1}^{n+1}=\left(d_{l p}^{S}\right)$ are characteristic matrices (for waves $\mathrm{P}$ and SV) of the entire environment and the environment under the source respectively.

From the matrix equation (13) we obtain

$$
U_{r 0}^{(1)}=\sum_{p=1}^{4} \frac{d_{12} d_{3 p}^{S}-d_{32} d_{1 p}^{S}}{d_{32} d_{11}-d_{31} d_{12}} s_{p}^{(1)} ; \quad U_{z 0}^{(1)}=\sum_{p=1}^{4} \frac{d_{31} d_{1 p}^{S}-d_{11} d_{3 p}^{S}}{d_{32} d_{11}-d_{31} d_{12}} s_{p}^{(1)} .
$$

Considering the second equality from formulas (11) and (12), and having done similar transformations, we obtain

$$
U_{r 0}^{(2)}=\sum_{p=1}^{4} \frac{d_{12} d_{3 p}^{s}-d_{32} d_{1 p}^{s}}{d_{32} d_{11}-d_{31} d_{12}} s_{p}^{(2)} ; \quad U_{z 0}^{(2)}=\sum_{p=1}^{4} \frac{d_{31} d_{1 p}^{s}-d_{11} d_{3 p}^{s}}{d_{32} d_{11}-d_{31} d_{12}} s_{p}^{(2)},
$$

where $s_{p}^{(2)}$ are the elements of the column vector $\mathbf{S}^{(2)}=\frac{1}{\eta^{2}}\left(1,1,-\frac{1}{\beta_{s}}, \frac{1}{\beta_{s}}\right)^{\mathrm{T}}$. 


\section{Calculation of Wave Fields in a Layered Half-space with Absorption based on the ...}

For the description of the spreading of SH waves, on the basis of the third equations from formulas (11) and (12) we obtain the relation

$$
\mathbf{Z}_{n+1}^{*}=\mathbf{D}_{s+1}^{n+1 *} \mathbf{S}^{*}+\mathbf{D}^{*} \mathbf{W}_{1}^{*}(0)
$$

where $\mathbf{D}_{s+1}^{* n+1}=\mathbf{A}_{n+1}^{*-1} \mathbf{C}_{n}^{*} \ldots \mathbf{C}_{s+2}^{*} \mathbf{A}_{s+1}^{*} \mathbf{L}_{s+1}^{*}, \mathbf{D}=\mathbf{A}_{n+1}^{*-1} \mathbf{C}_{n}^{*} \ldots \mathbf{C}_{1}^{*},\left(\mathbf{C}_{i}^{*}=\mathbf{A}_{i}^{*} \mathbf{L}_{i}^{*} \mathbf{A}_{i}^{*-1}\right), \mathbf{S}^{*}=\frac{\beta_{s}^{2}-1}{\beta_{s} \eta^{2}}(-1,1)^{\mathrm{T}}$, $\mathbf{W}_{1}^{*}(0)=\left(U_{\phi 0}, T_{z \phi 0}\right)^{\mathrm{T}}, \mathbf{Z}_{n+1}^{*}=\left(0, Z_{n+1}^{-}\right)^{\mathrm{T}}$. The matrices $\mathbf{D}^{*}=\left(d_{l p}^{*}\right)$ and $\mathbf{D}_{s+1}^{* n+1}=\left(d_{l p}^{*}\right)$ are characteristic matrices (for SH waves) of the entire environment and the environment under the source respectively. From the matrix equation (16) we obtain

$$
U_{\phi 0}=-\sum_{p=1}^{4} \frac{d_{1 p}^{*} s}{d_{11}^{*}} s_{p}^{*}
$$

If the source is at the depth $z_{s}>\sum_{i=1}^{n} h_{i}$ (inside of the half-space), then $\mathbf{D}_{s+1}^{n+1}$ and $\mathbf{D}_{s+1}^{* n+1}$ will be singular matrix of the order 4 and 2, respectively.

Described computational scheme, based on the Thomson-Haskell approach, for the case of the P-SV waves, leads to significant errors at high frequencies in the region of the boundary angles of wave spreading. The first reason for this is the bad stipulation of the matrix $\mathbf{D}$,resulting in a decreasing of the accuracy when calculating a minor $D_{12}^{13}=\left|\begin{array}{ll}d_{11} & d_{12} \\ d_{31} & d_{32}\end{array}\right|$, which is a denominator of expressions (14), (15) [1, $2,9]$.

The second reason for the numerical instability is the way of calculating of numerators of the expressions (14) and (15). In order to show this, we will write the characteristic matrix of the environment $\mathbf{D}$ as a product of characteristic matrices of the environment under the source $\mathbf{D}_{s+1}^{n+1}$ and over the source $\mathbf{D}_{1}^{s}$, that is $\mathbf{D}=\mathbf{D}_{s+1}^{n+1} \mathbf{D}_{1}^{s}$. Then, by expressing the elements of the matrix $\mathbf{D}$ in the functions (14) and (15) through the elements $d_{l p}^{1}$ of the matrix $\mathbf{D}_{1}^{s}$ and through the elements $d_{l p}^{s}$ of the matrix $\mathbf{D}_{s+1}^{n+1}$, we obtain

$$
U_{r 0}^{(1)}=\sum_{p=1}^{4} \frac{a_{r p}}{D_{12}^{13}} s_{p}^{(1)}, U_{z 0}^{(1)}=\sum_{p=1}^{4} \frac{a_{z p}}{D_{12}^{13}} s_{p}^{(1)}, U_{r 0}^{(2)}=\sum_{p=1}^{4} \frac{a_{r p}}{D_{12}^{13}} s_{p}^{(2)}, U_{z 0}^{(2)}=\sum_{p=1}^{4} \frac{a_{z p}}{D_{12}^{13}} s_{p}^{(2)},
$$

where

$$
\begin{gathered}
a_{r 1}=-d_{22}^{1} D_{12}^{s 13}-d_{32}^{1} D_{13}^{s 13}-d_{42}^{1} D_{14}^{s 13} ; a_{z 1}=d_{21}^{1} D_{12}^{s 13}+d_{31}^{1} D_{13}^{s 13}+d_{41}^{1} D_{14}^{s 13} ; \\
a_{r 2}=d_{12}^{1} D_{12}^{s 13}-d_{32}^{1} D_{23}^{s 13}-d_{42}^{1} D_{24}^{s 13} ; a_{z 2}=-d_{11}^{1} D_{12}^{s 13}+d_{31}^{1} D_{23}^{s 13}+d_{41}^{1} D_{24}^{s 13} ; \\
a_{r 3}=d_{12}^{1} D_{13}^{s 13}+d_{22}^{1} D_{23}^{s 13}-d_{42}^{1} D_{34}^{s 13} ; a_{z 3}=-d_{11}^{1} D_{13}^{s 13}-d_{21}^{1} D_{23}^{s 13}+d_{41}^{1} D_{34}^{s 13} ; \\
a_{r 4}=d_{12}^{1} D_{14}^{s 13}+d_{22}^{1} D_{24}^{s 13}+d_{32}^{1} D_{34}^{s 13} ; a_{z 4}=-d_{11}^{1} D_{14}^{s 13}-d_{21}^{1} D_{24}^{s 13}-d_{31}^{1} D_{34}^{s 13} ; \\
D_{l p}^{s i k}=\left|\begin{array}{ll}
d_{i l}^{s} & d_{i p}^{s} \\
d_{k l}^{s} & d_{k p}^{s}
\end{array}\right| \text { is the minor of matrix } \mathbf{D}_{s+1}^{n+1} .
\end{gathered}
$$

When calculating the minors $D_{12}^{13}$ and $D_{l p}^{s i k}$, large but roughly identical numbers are subtracted and the result may be outside the computer's bit network, or after the subtraction very few valid numerals may remain. These disadvantages of the Thomson-Haskell method can be eliminated by introducing other algorithms for calculating the minors. 


\section{Ruslan Pak, Olena Lanets}

Method of numerical stability increasing.

For every matrix $\mathbf{F}$ of the $4^{\text {th }}$ order can be put in compliance the matrix $\tilde{\mathbf{F}}$ of the $6^{\text {th }}$ order:

$$
\tilde{\mathbf{F}}=\left(\begin{array}{llllll}
F_{13}^{13} & F_{24}^{13} & F_{14}^{13} & F_{23}^{13} & F_{12}^{13} & F_{34}^{13} \\
F_{13}^{24} & F_{24}^{24} & F_{14}^{24} & F_{23}^{24} & F_{12}^{24} & F_{34}^{24} \\
F_{13}^{14} & F_{24}^{14} & F_{14}^{14} & F_{23}^{14} & F_{12}^{14} & F_{34}^{14} \\
F_{13}^{23} & F_{24}^{23} & F_{14}^{23} & F_{23}^{23} & F_{12}^{23} & F_{34}^{23} \\
F_{13}^{12} & F_{24}^{12} & F_{14}^{12} & F_{23}^{12} & F_{12}^{12} & F_{34}^{12} \\
F_{13}^{34} & F_{24}^{34} & F_{14}^{34} & F_{23}^{34} & F_{12}^{34} & F_{34}^{34}
\end{array}\right),
$$

elements of which are the minors of the $2^{\text {nd }}$ order of the matrix $\mathbf{F}$. By the rule (18) for the matrices $\mathbf{A}$, $\mathbf{A}^{-1}, \mathbf{C}$, we create the minor matrices $\tilde{\mathbf{A}}, \tilde{\mathbf{A}}^{-1}, \tilde{\mathbf{C}}$. Next, we are using the theorem: if, for any matrices of the fourth order $\mathbf{A}, \mathbf{B}$, и $\mathbf{C}$, for which $\mathbf{A} \mathbf{B}=\mathbf{C}$, we construct matrices $\tilde{\mathbf{A}}, \tilde{\mathbf{B}}$ and $\tilde{\mathbf{C}}$ using the formula (19), then equality $\tilde{\mathbf{A}} \tilde{\mathbf{B}}=\tilde{\mathbf{C}}$ will take place [2]. Thus, we obtain:

$$
\begin{gathered}
\tilde{\mathbf{D}}=\tilde{\mathbf{A}}_{n+1}^{-1} \tilde{\mathbf{C}}_{n} \ldots \tilde{\mathbf{C}}_{1} \quad\left(\tilde{\mathbf{C}}_{i}=\tilde{\mathbf{A}}_{i} \tilde{\mathbf{L}}_{i} \tilde{\mathbf{A}}_{i}^{-1} \text { matrix of layer } i\right) ; \\
\tilde{\mathbf{D}}_{s+1}^{n+1}=\tilde{\mathbf{A}}_{n+1}^{-1} \tilde{\mathbf{C}}_{n} \ldots \tilde{\mathbf{C}}_{s} \tilde{\mathbf{A}}_{s} .
\end{gathered}
$$

Replacing the minors in formulas (18) by the appropriate elements of the matrices (20), we obtain:

$$
U_{r 0}^{(1)}=\sum_{p=1}^{4} \frac{a_{r p}}{\tilde{d}_{15}} s_{p}^{(1)}, U_{z 0}^{(1)}=\sum_{p=1}^{4} \frac{a_{z p}}{\tilde{d}_{15}} s_{p}^{(1)}, U_{r 0}^{(2)}=\sum_{p=1}^{4} \frac{a_{r p}}{\tilde{d}_{15}} s_{p}^{(2)}, U_{z 0}^{(2)}=\sum_{p=1}^{4} \frac{a_{z p}}{\tilde{d}_{15}} s_{p}^{(2)},
$$

where

$$
\begin{aligned}
& a_{r 1}=-d_{22}^{1} \tilde{d}_{15}^{s}-d_{32}^{1} \tilde{d}_{11}^{s}-d_{42}^{1} \tilde{d}_{13}^{s}, a_{z 1}=d_{21}^{1} \tilde{d}_{15}^{s}+d_{31}^{1} \tilde{d}_{11}^{s}+d_{41}^{1} \tilde{d}_{13}^{s}, \\
& a_{r 2}=d_{12}^{1} \tilde{d}_{15}^{s}-d_{32}^{1} \tilde{d}_{14}^{s}-d_{42}^{1} \tilde{d}_{12}^{s}, a_{z 2}=-d_{11}^{1} \tilde{d}_{15}^{s}+d_{31}^{1} \tilde{d}_{14}^{s}+d_{41}^{1} \tilde{d}_{12}^{s}, \\
& a_{r 3}=d_{12}^{1} \tilde{d}_{11}^{s}+d_{22}^{1} \tilde{d}_{14}^{s}-d_{42}^{1} \tilde{d}_{16}^{s}, a_{z 3}=-d_{11}^{1} \tilde{d}_{11}^{s}-d_{21}^{1} \tilde{d}_{14}^{s}+d_{41}^{1} \tilde{d}_{16}^{s}, \\
& a_{r 4}=d_{12}^{1} \tilde{d}_{13}^{s}+d_{22}^{1} \tilde{d}_{12}^{s}+d_{32}^{1} \tilde{d}_{16}^{s}, a_{z 4}=-d_{11}^{1} \tilde{d}_{13}^{s}-d_{21}^{1} \tilde{d}_{12}^{s}-d_{31}^{1} \tilde{d}_{16}^{s} .
\end{aligned}
$$

To obtain the explicit expressions for the components of displacements on a free boundary, we must substitute formulas (21) into (10), and then the resulting expressions should be substituted into transformations (6).

To take into account the absorption, we set the complex velocities of the waves of the $\mathrm{P}$ and $\mathrm{S}$ type, assuming that the $Q$ factor does not depend on the frequency $\omega$. For a model of a environment with constant $Q$ complex velocities determined by the formula [7]

$$
v(\omega)=v_{0}\left(1+\frac{1}{\pi Q} \ln \frac{\omega}{2 \pi}\right)\left(1-\frac{j}{2 Q}\right)^{-1},
$$

where $v_{0}$ is the given velocity, $Q$ is the quality factor of the medium. Q-values may be different for waves of different types.

Modelling of local resonance effect.

The developed method gives the possibility to get a full wave field of P-SV type or SH type, including multiple, exchange, diffracted and other waves, and surface waves also.

Let us apply the described algorithm for calculating of synthetic seismograms while simulating a local resonance effect on the earth's surface. This phenomenon can be observed during the earthquakes (for example, the earthquake in Latour (India) in 1993 [10]) and it should be taken into account during the design of buildings. Dangerous seismic consequences are possible for buildings, own frequencies of which are in the local resonance band. 


\section{Calculation of Wave Fields in a Layered Half-space with Absorption based on the ...}

We consider a simple but realistic model of the environment (Table 1) to calculate synthetic seismograms [10]. In this model, the low-speed layer of soil $5 \mathrm{~m}$ thick is located on a basalt high-speed layer of thickness $300 \mathrm{~m}$. The point source in the form of force is located at a depth of $3 \mathrm{~km}$ in the granite half-space.

Table 1

Model of horizontal-layered medium [10]

\begin{tabular}{|c|c|c|c|c|c|c|}
\hline Number of the layer or half-space & $\rho, \mathrm{kg} / \mathrm{m}^{3}$ & $v_{P}, \mathrm{~m} / \mathrm{sec}$ & $v_{S}, \mathrm{~m} / \mathrm{sec}$ & $Q_{P}$ & $Q_{S}$ & $h, \mathrm{~m}$ \\
\hline 1 & 1300 & 1200 & 200 & 80 & 20 & 5 \\
\hline 2 & 2500 & 4500 & 2600 & 500 & 220 & 300 \\
\hline 3 & 2700 & 6000 & 3500 & 800 & 270 & $\infty$ \\
\hline
\end{tabular}

In Fig. 1 a synthetic seismogram calculated at an epicenter distance of $3 \mathrm{~km}$ for the environment model from Table. 1 is on the left, and for another environment model in which the upper soft layer is absent and the first layer with a thickness of $305 \mathrm{~m}$ has the same properties as the second layer from the Table 1 is on the right side. From the figure it is clear that the presence of soft soil layer leads to a significant increase of horizontal oscillations, and oscillations caused by S-waves are almost harmonic. On tangential component it is a P-wave of weak intensity due to the wave field in the near-field region.

In Fig. 2 the amplitude spectra of synthetic seismograms are given. The value of their horizontal components at a resonant frequency of $10.5 \mathrm{~Hz}$ (in the presence of a soft layer) is several times larger than the appropriate components in the absence of a soft layer. At the same time, the presence or absence of a layer of reduced velocities slightly affects on the vertical component of oscillations. This way, the lowspeed top layer of the soil may cause the local oscillation amplification effects.

Therefore, the synthetic seismograms can be used to estimate both the resonant frequency and the coefficient of soil oscillations amplification.

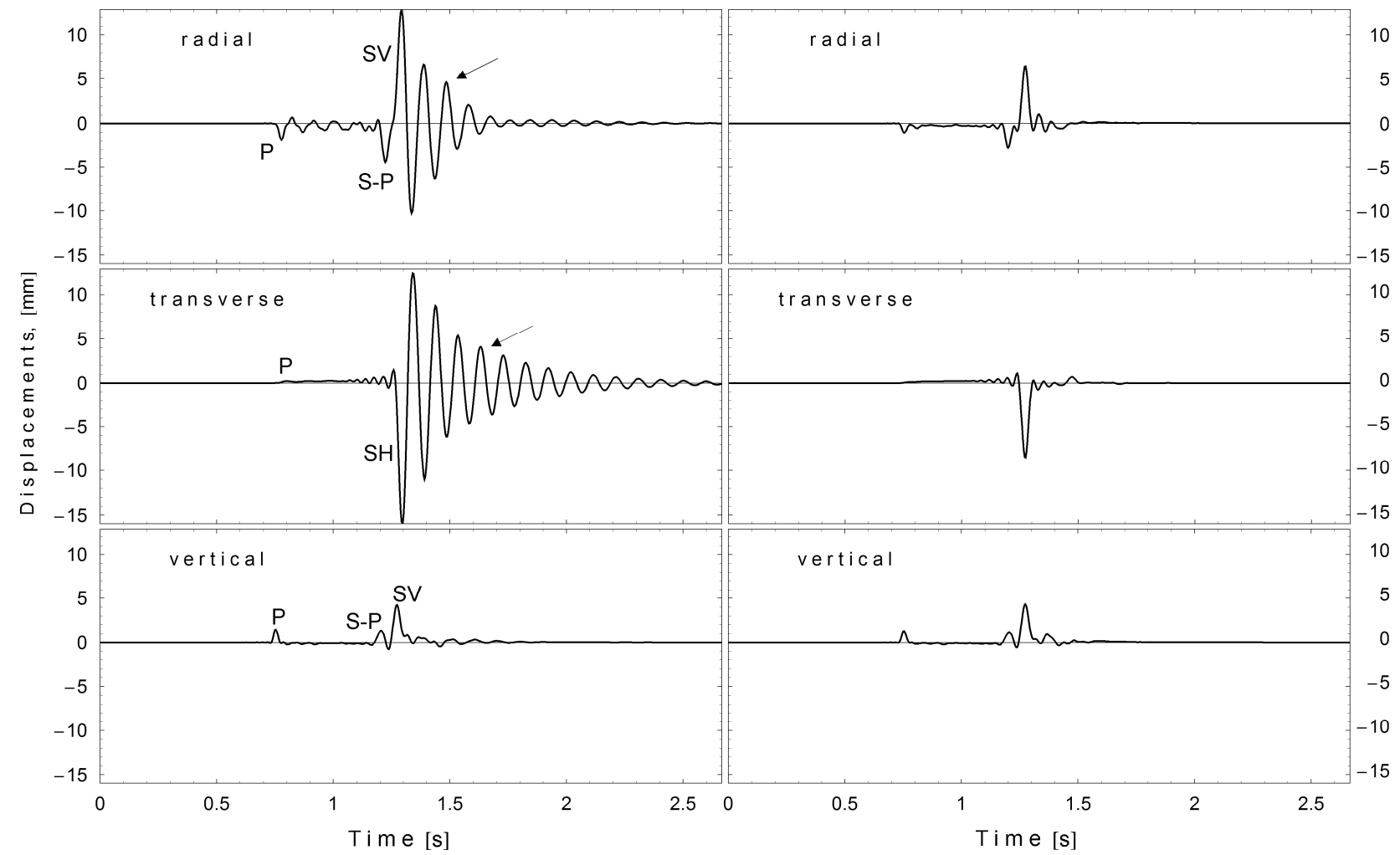

Fig. 1. Comparison of synthetic seismograms for a medium model with a soft layer on the surface (left) and without a soft layer (right). Seismograms are calculated for a point source in the form of a force located at a depth of $3 \mathrm{~km}$, an epicentre distance of $3 \mathrm{~km}$ and azimuth of $90^{\circ}$. The time dependence of the source is described by the function $1 / \tau \sin ^{3}(\pi t / \tau)$, if, $0 \leq t \leq \tau$, otherwise 0 , where $\tau=50 \mathrm{~ms}$ 


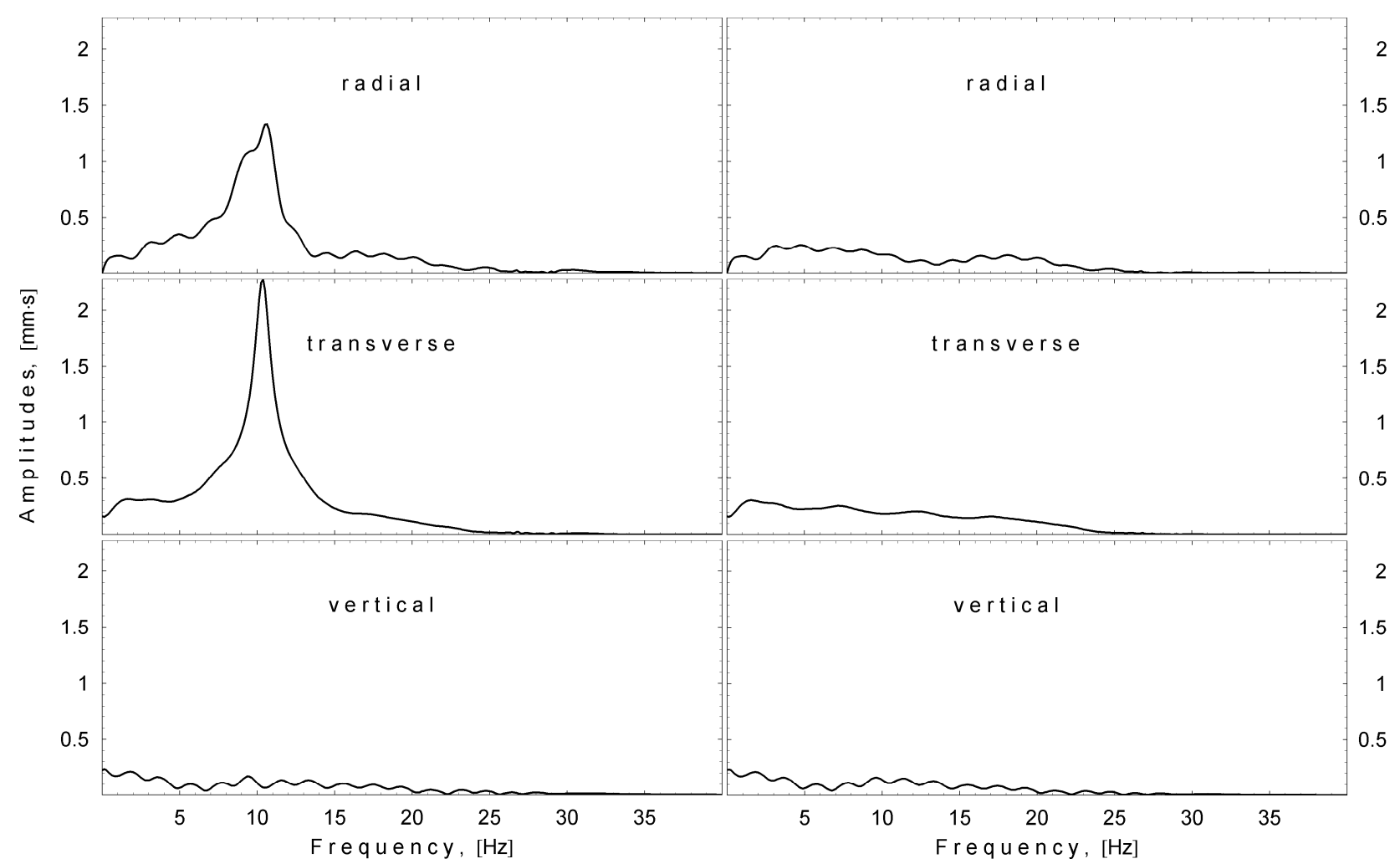

Fig. 2. Amplitude spectra of synthetic seismograms, shown in Fig. 1 for an impulse source, if $\tau \rightarrow 0$

\section{Conclusions}

On the basis of the Thomson-Haskell matrix method, an effective and stable method for calculating a seismic wave field for a multi-layer horizontal-layered isotropic environment with absorption has been developed. The method takes into account the presence of free surface, the presence of a point source in the form of simple force, interference phenomena associated with thin-layering. Transition from characteristic matrices of the fourth order to the matrices of the sixth order was made to increase the stability of the wave field calculation. The results are demonstrated on the example of simulation of the resonance phenomenon in a horizontal-layered environment associated with the presence of the upper lowspeed layer.

\section{References}

[1] L.A. Molotkov, Matrychnyi metod v teoii rasprostranenija voln $v$ sloystykh, upruhikh i zhydkikh sredakh ["The matrix method in the theory of wave propagation in layered, elastic and liquid environment."]. Leningrad, Russia: Nauka Publ., 1984. [in Russian].

[2] J.W. Dunkin, " Computation of modal solution in layered elastic media at high frequencies," Bulletin of the Seismological Society of America, vol. 55, no. 2, pp. 335-358, 1965.

[3] A. Abo-Zena, "Dispersion function computations for unlimited frequency values," Geophys. J. R. Astron. Soc., vol. 58, no. 1, pp. 91-105, Jul. 1979.

[4] B. Kennet, Seismic Wave Propagation in Stratified Media. Canberra, Australia: ANU E Press, 2011.

[5] G. Muller, "The reflectivity method: a tutorial," Journal of Geophysics, vol. 58, issue 1-3, pp. 153-174, 1985.

[6] M. Bouchon, "A review of the discrete wavenumber method," Pure Appl. Geophys., vol. 160, no. 3-4, pp. $445-465,2003$.

[7] K. Aki, and P. Richards, Quantitative seismology. Sausalito, CA: University Science Book, 2002.

[8] Yu. V. Rohanov, R.M. Pak "Predstavlenie potentsiala ot tochechnykh istochnikov dlia odnorodnoi izotropnoi sredy v vide intehralov Besselia-Mellina" ["Representation of potential from point sources for a homogeneous isotropic medium in the form of Bessel-Mellin integrals"], Heofyzychnyi zhurnal [Journal of Geophysics], vol. 35, no 2., pp. 163-167, 2013. [in Russian]. 
Calculation of Wave Fields in a Layered Half-space with Absorption based on the ...

[9] L. Knopoff, “A matrix method for elastic wave problem," Bulletin of the Seismological Society of America, vol. 55, no. 1, pp. 431-438, 1964.

[10] M. Baumbach, etc., "Study of the Foreshocks and Aftershocks of the Intraplate Latur Earthquake of September 30, 1993, India", in Latur earthquake, (Memoir / Geological Society of India; 35), Geological Society of India, pp. 33-63, 1994. 